\title{
Measuring the momentum of a nanomechanical oscillator through the use of two tunnel junctions
}

\author{
C.B. Doiron, ${ }^{1}$ B. Trauzettel, ${ }^{2,1}$ and C. Bruder ${ }^{1}$ \\ ${ }^{1}$ Department of Physics, University of Basel, CH-4056 Basel, Switzerland \\ ${ }^{2}$ Institute of Theoretical Physics and Astrophysics, \\ University of Würzburg, D-97074 Würzburg, Germany
}

(Dated: November 2007)

\begin{abstract}
We propose a way to measure the momentum $p$ of a nanomechanical oscillator. The $p$-detector is based on two tunnel junctions in an Aharonov-Bohm-type setup. One of the tunneling amplitudes depends on the motion of the oscillator, the other one not. Although the coupling between the detector and the oscillator is assumed to be linear in the position $x$ of the oscillator, it turns out that the finite-frequency noise output of the detector will in general contain a term proportional to the momentum spectrum of the oscillator. This is a true quantum phenomenon, which can be realized in practice if the phase of the tunneling amplitude of the detector is tuned by the AharonovBohm flux $\Phi$ to a $p$-sensitive value.

PACS numbers: $72.70 .+\mathrm{m}, 73.23 .-\mathrm{b}, 85.85 .+\mathrm{j}$
\end{abstract}

In nano-electromechanical (NEM) systems, it is the position of the oscillator that typical measurement devices (like tunnel junctions or single electron transistors) are coupled to. Using these detectors, position measurements with sensitivities close to the standard quantum limit have already been observed [1, 2, 3]. From a fundamental point of view, it is desirable to go further, i.e. to prepare and manipulate NEM oscillators in the quantum regime. A quantum NEM system would allow us to study an ideal realization of a continuous variable quantum system [4]. The exploration of such systems has to be seen as complementary to the wide study of two-level systems done in the context of quantum computing.

In order to be able to fully characterize a continuous variable quantum system that is described by two noncommuting operators $\hat{x}$ and $\hat{p}$, we need to be able to measure expectation values of moments of both of them [5]. Only this allows, for instance, to detect the entanglement between two (or more) NEM devices [6]. The literature already contains proposals regarding quantum measurements of the momentum of macroscopic objects like those used for gravity-wave detection [7]. However, none of these proposals have been realized in practice. In this Letter, we propose a realistic and feasible way to measure the momentum of a nanometer-sized resonator. This is a nontrivial task since the coupling between the detector and the oscillator is naturally described by an $x$-dependence but not a $p$-dependence. Nevertheless, the proposed setup (shown in Fig. 1b) allows for a measurement of the momentum spectrum $S_{p}(\omega)=\int d t e^{i \omega t}\langle\{\hat{p}(t), \hat{p}(0)\}\rangle$ of the oscillator. This can be done because we have found a way to tune the phase of the tunnel coupling term that is sensitive to the position of the oscillator by an Aharonov-Bohm (AB) flux $\Phi$, see Fig. 10. Related setups have been investigated recently in the context of dephasing due to the coupling of an $\mathrm{AB}$ ring structure to a NEM device [8]. A typical position detec- tor which has been analyzed theoretically in great detail 9, 10, 11, 12, 13, 14, 15] and experimentally realized 16, 17] is depicted in Fig. 17. It shows a single tunnel junction coupled to a NEM oscillator. A thorough analysis of the coupled quantum system leads to the result that the output signal of the detector is sensitive to the position spectrum $S_{x}(\omega)=\int d t e^{i \omega t}\langle\{\hat{x}(t), \hat{x}(0)\}\rangle$ of the oscillator. The modification of the detector shown in Fig. 1b instead allows for a measurement of $S_{p}(\omega)$.

The Hamiltonian of the coupled system $H=H_{\mathrm{osc}}+H_{B}+$ $H_{\text {tun }}$ is the sum of the Hamiltonian of the (quantum) harmonic oscillator $H_{\mathrm{osc}}$ (with mass $M$ and frequency $\Omega$ ), the bath Hamiltonian $H_{B}$ (describing the leads of the detector), and the tunneling Hamiltonian $H_{\text {tun }}$ (which couples the dynamics of the electrons that tunnel across the junction to the motion of the oscillator):

$$
\begin{aligned}
H_{\mathrm{osc}} & =\hbar \Omega\left(a^{\dagger} a+\frac{1}{2}\right)=\frac{\hat{p}^{2}}{2 M}+\frac{M \Omega^{2} \hat{x}^{2}}{2} \\
H_{B} & =\sum_{k} \varepsilon_{k} c_{k}^{\dagger} c_{k}+\sum_{q} \varepsilon_{q} c_{q}^{\dagger} c_{q}, \\
H_{\mathrm{tun}} & =T(\hat{x}) Y^{\dagger} \sum_{k, q} c_{k}^{\dagger} c_{q}+T^{\dagger}(\hat{x}) Y \sum_{k, q} c_{q}^{\dagger} c_{k} .
\end{aligned}
$$

Here, $k(q)$ is a wave-vector in the right (left) lead, $c^{(\dagger)}$ is the electron annihilation (creation) operator, and $Y^{(\dagger)}$ is an operator that decreases (increases) $m$, the number of electrons that have tunneled through the system, by one. It allows one to keep track of the transport processes during the evolution of the system. For typical nanoresonators, the mass $M$ varies between $10^{-18}$ and $10^{-15} \mathrm{~kg}$, and the resonance frequency is usually $1 \mathrm{MHz}<\Omega / 2 \pi<1 \mathrm{GHz}[18]$.

We first discuss the model in the standard configuration shown in Fig. 17 and later on describe the new setup in Fig. 1b. For small displacements with respect to the tunneling length (which is the relevant regime in typi- 
a

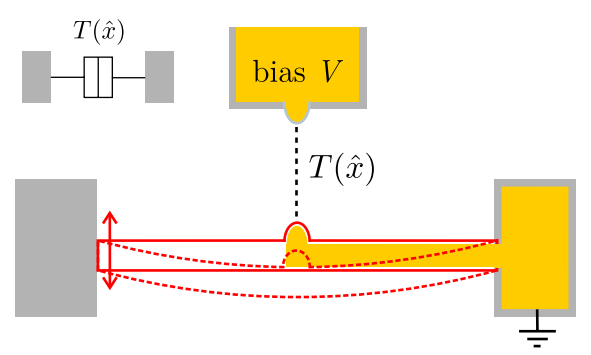

b

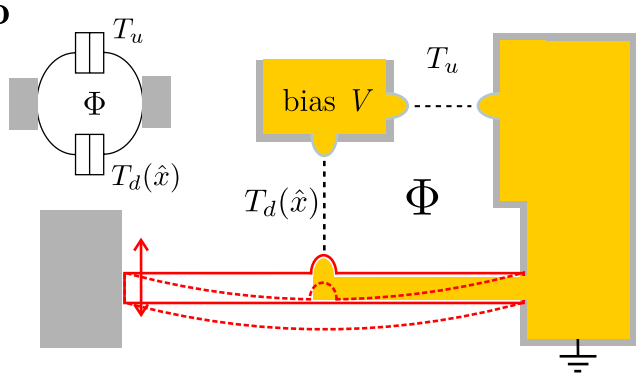

FIG. 1: (color online) a Position detector. The figure shows schematically a position detector of the motion of a NEM oscillator (red). The detector is based on a tunnel junction with a tunnel matrix element $T(\hat{x})$ which depends on the position of the oscillator. The shaded regions (yellow) are assumed to be conducting. b Position and/or momentum detector. The figure illustrates a detector which contains two tunnel junctions that form a loop threaded by a magnetic flux $\Phi$. Tuning the flux will change the performance of the detector from being able to detect the power spectrum of the position operator of the oscillator to being able to detect the power spectrum of the momentum operator of the oscillator. For clarity, the two insets show a simplified illustration of the detectors in $\mathbf{a}$ and $\mathbf{b}$.

cal experiments on NEM devices), the tunneling amplitude $T(\hat{x})$ can be taken as a linear function of $\hat{x}$, namely $T(\hat{x})=\left(e^{i \varphi_{0}} / 2 \pi \Lambda\right)\left(\tau_{0}+e^{i \eta} \tau_{1} \hat{x}\right)$, where $\tau_{0}$ and $\tau_{1}$ are real, and $\Lambda$ is the density of states. The phases $\varphi_{0}$ and $\eta$ describe details of the detector-oscillator coupling [19]. It can be shown that our detector is quantum-limited for any $\eta$, according to the definition of a quantum-limited detector in Ref. [14]. Within the single-junction setup (Fig. 1 1 ), the relative phase $\eta$ is sample-dependent and cannot be tuned experimentally. In a typical device, the $x$-dependence of the phase of $T(\hat{x})$ is much weaker than the $x$-dependence of the amplitude. Then, we can set $\eta \simeq 0$, and the tunnel junction acts as a positionto-current amplifier where the frequency-dependent current noise $S_{I}(\omega)$ of the detector contains a term proportional to the position spectrum $S_{x}(\omega)$ of the oscillator, i.e. $\Delta S_{I}(\omega)=S_{I}(\omega)-2 e\langle I\rangle \approx \lambda_{x}^{2} S_{x}(\omega)$ with $\lambda_{x}$ the gain of the amplifier [13, 20].

We now demonstrate that a tunnel junction with a phase $\eta=\pi / 2 \bmod \pi$ acts as a momentum detector and $\Delta S_{I}(\omega) \approx \lambda_{p}^{2} S_{p}(\omega)$, where $\lambda_{p}$ is the gain of the momentum-to-current linear amplifier. The critical requirement to build a momentum detector is to be able to vary $\eta$ experimentally. This can be done using the AB-type setup shown in Fig. 1b: a metallic ring where one arm is a standard tunnel junction position detector with tunneling amplitude $T_{d}(\hat{x})$, and the other arm is a position-independent tunnel junction with tunneling amplitude $T_{u}$ 21]. The total transmission amplitude $T(\hat{x}, \Phi)$ of the device is the sum of both tunneling amplitudes [23]. Since only one arm shows a position-dependence, the induced phase difference between the two arms affects the position-independent and the position-dependent parts of the tunneling amplitudes $\tau_{0}$ and $\tau_{1}$ in a different way. Explicit calculation shows that the tunneling amplitude is given (up to a global gauge-dependent phase factor) by

$$
\begin{aligned}
T(\hat{x}, \Phi) & =\left(\tau_{0}(\Phi)+e^{i \eta(\Phi)} \tau_{1} \hat{x}\right) /(2 \pi \Lambda) \text { with } \\
\tau_{0}^{2}(\Phi) & =\tau_{0, d}^{2}+\tau_{0, u}^{2}+2 \tau_{0, d} \tau_{0, u} \cos \left(2 \pi \frac{\Phi}{\Phi_{0}}+\varphi_{0, d}-\varphi_{0, u}\right) \\
\eta(\Phi) & =2 \pi \frac{\Phi}{\Phi_{0}}+\varphi_{1, d}-\varphi_{0, u} \\
& -\operatorname{Arg}\left(\tau_{0, u}+e^{i\left(2 \pi \frac{\Phi}{\Phi_{0}}+\varphi_{0, d}-\varphi_{0, u}\right)} \tau_{0, d}\right)
\end{aligned}
$$

where we have defined $T_{u} \equiv e^{i \varphi_{0}, u} \tau_{0, u}, T_{d} \equiv e^{i \varphi_{0}, d} \tau_{0, d}+$ $e^{i \varphi_{1, d}} \tau_{1, d} \hat{x}, \tau_{1} \equiv \tau_{1, d}$, and $\Phi_{0}=h / e$ [24]. The positionindependent part of the tunneling amplitude $\tau_{0}(\Phi)$ displays the standard $\mathrm{AB}$ oscillations as a function of flux. Likewise, the relative phase $\eta(\Phi)$ shows a distinct dependence on the flux. Importantly, for $\tau_{0, u}>\tau_{0, d}$, the phase $\eta(\Phi)$ can be tuned continuously in the whole range $[-\pi, \pi]$. In the limit, where $\tau_{0, u} \gg \tau_{0, d}, \eta(\Phi) \sim$ $2 \pi \frac{\Phi}{\Phi_{0}}+\eta(\Phi=0)$ varies linearly with the applied flux. In the opposite regime $\tau_{0, u} \ll \tau_{0, d}, \eta$ no longer depends on $\Phi$. Therefore, it is crucial to put the tunneling amplitudes in the regime where $\eta(\Phi)$ can be tuned to $\pi / 2$. We will show below that a feasible way to calibrate $\eta(\Phi)$ to the $p$-sensitive point $\pi / 2$ is a measurement of the flux dependence of the current through the $\mathrm{AB}$ detector.

Similarly to Refs. [13, 20], we study the coupled system using the quantum equation of motion for the chargeresolved density matrix within the Born-Markov approximation, assuming that $e V \gg \hbar \Omega$.

It has been derived previously that, under the assumption that the tunneling amplitude depends linearly on $\hat{x}$, the equation of motion for the reduced density matrix of the oscillator is of Caldeira-Leggett form [12, 13, 15]. Thus, it contains both a damping and a diffusion term. When the electron temperature is much smaller than the applied bias (and taking $V>0$ ), the detector-induced damping coefficient is $\gamma_{+}=\hbar \tau_{1}^{2} /(4 \pi M)$ and the diffusion coefficient is $D_{+}=2 M \gamma_{+} k_{B} T_{\text {eff }}$ with $T_{\text {eff }}=e V / 2 k_{B}$. 
In general, the oscillator is not only coupled to the detector but also to the environment. The coupling to this additional bath is controlled via $\gamma_{0}=\Omega / Q_{0}$ (related to the finite quality factor $Q_{0}$ of the mode, which in current experiments varies from $10^{3}$ to $10^{6}$ [18] ) and the associated diffusion constant $D_{0}=2 M \gamma_{0} k_{B} T_{\text {env }}$ that must be added to the detector-induced damping and diffusion constants to find the total damping coefficient $\gamma_{\text {tot }}=\gamma_{+}+\gamma_{0}$ and the total diffusion coefficient $D_{\text {tot }}=D_{0}+D_{+} \cdot T_{\text {env }}$ denotes the temperature of the environment. In typical experiments, it varies from $30 \mathrm{mK}$ to $10 \mathrm{~K}$. Within our model, all the these system parameters are independent of the applied flux.

It is now straightforward to calculate the current and the current noise of the detector. We skip the details here (see Ref. [20] for $\eta=0$ ) and directly turn to the results. The average current of the detector is given by

$$
\begin{aligned}
I & =\frac{e^{2} V}{h}\left(\tau_{0}^{2}+2 \cos \eta \tau_{0} \tau_{1}\langle x\rangle+\tau_{1}^{2}\left\langle x^{2}\right\rangle\right) \\
& -\frac{2 e \gamma_{+} \tau_{0}}{\hbar \tau_{1}} \sin \eta\langle p\rangle-e \gamma_{+} .
\end{aligned}
$$

For $\eta \neq 0 \bmod \pi$, the average current contains a term proportional to the average momentum of the oscillator that does not vary with the applied bias [15]. However, since $\langle p\rangle=0$ in the steady-state, the average current contains no information about the momentum of the oscillator. Therefore, the current of the detector can not be used as a $p$-detector in the steady-state. Nevertheless, the current is important to calibrate $\eta$ to the $p$-sensitive value $\pi / 2$. A careful analysis of the current $I$ as a function of $\Phi$ shows that the inflection points of $I(\Phi)$ correspond precisely to values of $\eta=\pi / 2 \bmod \pi$. Therefore, we can use a current measurement to tune $\eta$ to a $p$-sensitive value.

In the experimentally relevant regime, where $\tau_{1}^{2}\left\langle x^{2}\right\rangle \ll$ $\tau_{0}^{2}$, and for $\omega \sim \Omega$, the dominant contributions to the current power spectrum of the detector are

$$
\begin{aligned}
& S_{I}(\omega)=2 e\langle I\rangle+8 e^{2} \omega \int_{0}^{\infty} d t \sin (\omega t) \times \\
& {\left[\frac{e V}{h} \cos \eta \tau_{0} \tau_{1}\langle\langle x m\rangle\rangle-\frac{\gamma_{+} \tau_{0}}{\hbar \tau_{1}} \sin \eta\langle\langle p m\rangle\rangle\right],}
\end{aligned}
$$

where $\langle\langle a b\rangle\rangle=\langle a b\rangle-\langle a\rangle\langle b\rangle$. We now further analyze the added noise due to the presence of the oscillator, $\Delta S=S_{I}(\omega)-2 e\langle I\rangle$. This noise spectrum is the sum of a contribution arising due to correlations between the transfered charge $m$ and position (term $\sim\langle\langle x m\rangle\rangle$ in Eq. (6)), which we call $\Delta S_{1}$, and one due to correlations between $m$ and the momentum of the oscillator (term $\sim\langle\langle p m\rangle\rangle$ in Eq. (6) $)$, which we call $\Delta S_{2}$. The full spec- trum is therefore $\Delta S=\Delta S_{1}+\Delta S_{2}$ with

$$
\begin{aligned}
& \Delta S_{1}(\omega)=\left[\lambda_{x}^{2}\left(1-\frac{\hbar \Omega}{2 e V} \frac{\Delta x_{0}^{2}}{\left\langle\left\langle x^{2}\right\rangle\right\rangle}\right)\right. \\
& \left.-\lambda_{x} \lambda_{p}\left(\frac{M \Omega}{2 \pi} \tau_{1}^{2} \Delta x_{0}^{2} \frac{e V}{M \Omega^{2}\left\langle\left\langle x^{2}\right\rangle\right\rangle}\right)\right] S_{x}(\omega) \\
& -\lambda_{x} \lambda_{p}\left(1-\frac{M e V}{\left\langle\left\langle p^{2}\right\rangle\right\rangle}\right) \frac{\left\langle\left\langle p^{2}\right\rangle\right\rangle}{M} \frac{4\left(\Omega^{2}-\omega^{2}\right)}{4 \gamma_{\mathrm{tot}}^{2} \omega^{2}+\left(\omega^{2}-\Omega^{2}\right)^{2}} \\
& \Delta S_{2}(\omega)=\lambda_{p}^{2}\left(1-\frac{M e V}{\left\langle\left\langle p^{2}\right\rangle\right\rangle}\right) S_{p}(\omega) \\
& +\left[\lambda_{p} \lambda_{x}\left(1-\frac{\hbar \Omega}{2 e V} \frac{\Delta x_{0}^{2}}{\left\langle\left\langle x^{2}\right\rangle\right\rangle}\right)\right. \\
& \left.-\lambda_{p}^{2}\left(\frac{M \Omega}{2 \pi} \tau_{1}^{2} \Delta x_{0}^{2} \frac{e V}{M \Omega^{2}\left\langle\left\langle x^{2}\right\rangle\right\rangle}\right)\right] \frac{4 M \Omega^{2}\left\langle\left\langle x^{2}\right\rangle\right\rangle\left(\Omega^{2}-\omega^{2}\right)}{4 \gamma_{\mathrm{tot}}^{2} \omega^{2}+\left(\omega^{2}-\Omega^{2}\right)^{2}},
\end{aligned}
$$

where the position and the momentum gain are given by $\lambda_{x}=2 e \tau_{0} \tau_{1}(e V / h) \cos \eta$ and $\lambda_{p}=(e / 2 \pi M) \tau_{0} \tau_{1} \sin \eta$, respectively. We now discuss several limits of the current noise $S_{I}(\omega)$ of the detector in the case of a general phase $\eta$. For $\eta=0 \bmod \pi$, we recover Eq. (30) of Ref. 20] - the position detector result. More interestingly, for $\eta=\pi / 2 \bmod \pi, \lambda_{x}=0$ and the detector output contains only two terms: The first one is proportional to $S_{p}(\omega)$ and therefore peaked around $\Omega$. The second one is proportional to $\left(\Omega^{2}-\omega^{2}\right)$ and contributes negligibly near resonance $\omega \approx \Omega$. Hence, for $\eta=\pi / 2$, we obtain

$$
\Delta S(\omega \approx \Omega) \approx \lambda_{p}^{2}\left(1-\frac{M e V}{\left\langle\left\langle p^{2}\right\rangle\right\rangle}\right) S_{p}(\omega)
$$

Thus, the added noise is directly proportional to the momentum spectrum of the oscillator. This is the key result of our Letter.

From the parameter dependence of each gain, we can estimate that the momentum signal at $\eta=\pi / 2$ should be typically smaller than the position signal at $\eta=0$ by a factor $(\mathrm{eV} / \hbar \Omega)^{2}$. Nevertheless, it is unambiguously possible to identify a $p$ signal in the current noise. We now describe three different ways to do this. First, since $\lambda_{x} \propto V$ while $\lambda_{p}$ is independent of $V$, the bias voltage dependence of the noise spectrum can also be used to confirm that momentum fluctuations are measured. Secondly, for an oscillator undergoing Brownian motion, the temperature dependence of both signals differs qualitatively. Like in the position detector case, the momentum signal is reduced by a quantum correction (the term proportional to $-\mathrm{MeV} /\left\langle\left\langle p^{2}\right\rangle\right\rangle$ in Eq. (9)) that arises from the finite commutator of $\hat{x}$ and $\hat{p}$. However, there is a fundamental difference between the $x$-detector result (Eq. (7) of Ref. [13]) and the $p$-detector result (Eq. (9)). In the former case, the quantum corrections are always small compared to the leading terms and therefore the peak at resonance is always positive. In contrast, the two terms in Eq. (9) can be of equal magnitude and compete about the sign of $\Delta S(\omega \approx \Omega)$. The $p$-sensitive current noise in Eq. (9) 
changes sign when the effective temperature of the oscillator is equal to $\left(e V / k_{B}\right)\left(1-\gamma_{+} / 2 \gamma_{\text {tot }}\right) /\left(1-\gamma_{+} / \gamma_{\text {tot }}\right)$. For a cold environment $T_{\text {env }} \ll e V, \Delta S(\omega)$ is negative at the resonance, whereas, for a hot environment $T_{\text {env }}>\mathrm{eV}$, $\Delta S(\omega)$ is positive. This change of sign never appears during a position measurement, so this pronounced difference between a $x$-dependent and a $p$-dependent signal can be used distinguish the two. We illustrate the change of sign in the inset of Fig. 2, where the added current noise for $\eta=\pi / 2$ is plotted for different $T_{\text {env }}$ 25].

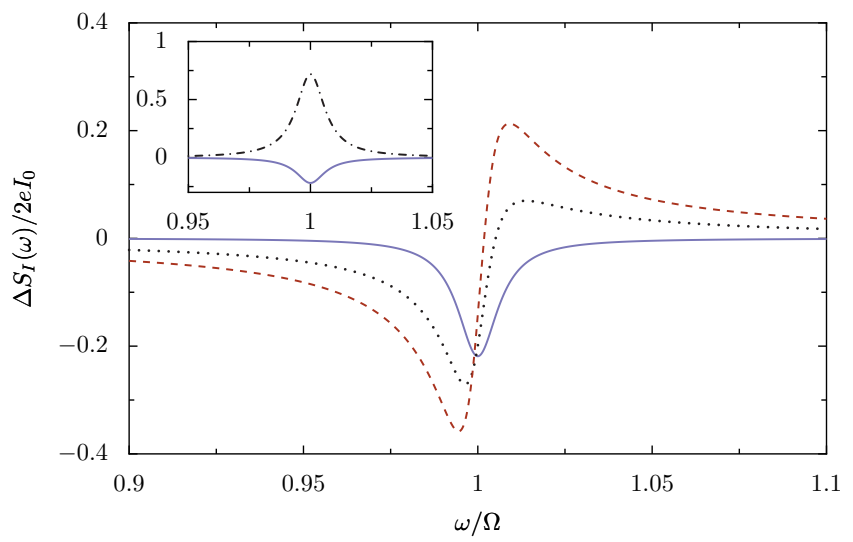

FIG. 2: (color online) Added current noise (normalized by $\left.2 e I_{0} \equiv 2 e^{3} \tau_{0}^{2} V / h\right)$ of the proposed momentum detector due to the presence of the oscillator. For all curves, the bias is $e V=$ $50 \hbar \Omega, \gamma_{+}=\gamma_{\text {tot }} / 4$ and $\gamma_{\text {tot }}=\Omega / 200$. The main panel shows the total detector output for different values of the tunneling phase $\eta$ and for $T_{\text {env }}=0$. The (blue) solid, (black) dotted, and (red) dashed lines correspond to $2 \eta / \pi=1,1.005$, and 1.01, respectively. In the inset, the current noise at the $p$-sensitive phase $\eta=\pi / 2$ is plotted for two different temperatures of the environment $T_{\mathrm{env}}=0\left(\right.$ solid line) and $T_{\mathrm{env}}=5 \mathrm{eV} / k_{B}$ (dash-dotted line).

In the main panel of Fig. 2, we plot the full detector output for different values of $\eta$ near the optimal operation point for momentum detection. Away from $\eta=\pi / 2$, contributions to the current noise $\sim \lambda_{x}^{2}$ become important and wash out the momentum signal $\sim \lambda_{p}^{2}$. Indeed, for small $\Delta \eta=\eta-\pi / 2$, the ratio $\lambda_{x} / \lambda_{p} \sim(-\Delta \eta)(e V / \hbar \Omega)$ of the two amplification factors becomes large as soon as $|\Delta \eta|>\hbar \Omega / e V$. In the high-bias regime $(e V \gg \hbar \Omega)$, momentum detection therefore requires good experimental control over the applied flux. At moderate bias $e V \geq \hbar \Omega$, the requirement on $\Delta \eta$ becomes less restrictive. Finally, the current noise spectrum at $\eta=\pi / 2$ shows a strong symmetry around $\Omega$ that makes the optimal operation point easily identifiable.

In conclusion, we have shown how a modified tunnel junction position detector can be designed to detect the momentum fluctuations of a NEM oscillator. By using two tunnel junctions in an AB-type setup, it is possible to precisely tailor the interaction Hamiltonian between the detector and the oscillator via an external magnetic field.
We have demonstrated how the proposed detector can be made sensitive to either displacement or momentum fluctuations of the oscillator.

We would like to thank W.A. Coish, L.I. Glazman, O. Gühne, and I. Martin for interesting discussions. This work was financially supported by the NSERC of Canada, the FQRNT of Québec, the Swiss NSF, and the NCCR Nanoscience.

[1] R. G. Knobel and A. N. Cleland, Nature 424, 291 (2003).

[2] M. LaHaye, O. Buu, B. Camarota, and K. Schwab, Science 304, 74 (2004).

[3] A. Naik et al., Nature 443, 193 (2006).

[4] S. L. Braunstein and P. van Loock, Rev. Mod. Phys. 77, 513 (2005).

[5] L.-M. Duan, G. Giedke, J. I. Cirac, and P. Zoller, Phys. Rev. Lett. 84, 2722 (2000).

[6] J. Eisert, M. B. Plenio, S. Bose, and J. Hartley, Phys. Rev. Lett. 93, 190402 (2004).

[7] V. B. Braginsky, Y. I. Vorontsov and K. S. Thorne, Science 209, 547 (1980); C. M. Caves et al., Rev. Mod. Phys. 52, 341 (1980); V. B. Braginsky and F. Y. Khalili, Phys. Lett. A 147251 (1990). V. B. Braginsky, M. L. Gorodetsky, F. Y. Khalili and K. S Thorne, Phys. Rev. D 61, 044002 (2000); V. B. Braginsky et al., Phys. Rev. D 67 082001 (2003).

[8] A. D. Armour and M. P. Blencowe, Phys. Rev. B 64, 035311 (2001); F. Romeo, R. Citro, and M. Marinaro, Phys. Rev. B 76, 081301(R) (2007).

[9] M. F. Bocko, K. A. Stephenson, and R. H. Koch, Phys. Rev. Lett. 61, 726 (1988).

[10] B. Yurke and G. P. Kochanski, Phys. Rev. B 41, 8184 (1990).

[11] N. F. Schwabe, A. N. Cleland, M. C. Cross, and M. L. Roukes, Phys. Rev. B 52, 12911 (1995).

[12] D. Mozyrsky and I. Martin, Phys. Rev. Lett. 89, 018301 (2002).

[13] A. A. Clerk and S. M. Girvin, Phys. Rev. B 70, 121303(R) (2004).

[14] A. A. Clerk, Phys. Rev. B 70, 245306 (2004).

[15] J. Wabnig, D.V. Khomitsky, J. Rammer, and A.L. Shelankov, Phys. Rev. B 72, 165347 (2005); J. Wabnig, J. Rammer, and A.L. Shelankov, Phys. Rev. B 75, 205319 (2007).

[16] A. N. Cleland, J. S. Aldridge, D. C. Driscoll, and A. C. Gossard, Appl. Phys. Lett. 81, 1699 (2002).

[17] N. E. Flowers-Jacobs, D. R. Schmidt, and K. W. Lehnert, Phys. Rev. Lett. 98, 096804 (2007).

[18] K.L. Ekinci and M.L. Roukes, Rev. Sci. Instr. 76, 061101 (2005).

[19] The overall phase $\varphi_{0}$ is a gauge-dependent quantity and does not affect the observable output of the detector.

[20] C. B. Doiron, B. Trauzettel, and C. Bruder, Phys. Rev. B 76, 195312 (2007).

[21] A similar device in a different context (without a positiondependent tunneling amplitude) has been realized in the electronic Mach-Zehnder interferometer 22].

[22] Y. Ji et al., Nature 422, 415 (2003).

[23] We assume that the size of the $\mathrm{AB}$ detector is smaller 
than the phase coherence length (which can be several microns in metallic thin films at low temperatures) and that it is large enough such that we do not have to take into account the change of the area of the AB loop due to the fluctuating position of the oscillator. Furthermore, the setup is designed such that the electrons can not make roundtrips in the AB loop but leave the ring instead after going either through the upper or the lower arm. This explains why (in general) $|T(\hat{x}, \Phi)|^{2} \neq|T(\hat{x},-\Phi)|^{2}[$ see
M. Büttiker, Phys. Rev. Lett. 57, 1761 (1986)].

[24] Strictly speaking, the analysis in Eq. (4) is only valid in the single-channel case. For the multi-channel case with $N$ channels in the AB loop, the magnitude of the $\mathrm{AB}$ oscillations is reduced by a factor $1 / N$.

[25] The plots are done for an oscillator in the thermal regime: $\left\langle\left\langle p^{2}\right\rangle\right\rangle=M k_{B} T_{\text {eff }}$ with $\gamma_{\text {tot }} T_{\text {eff }}=T_{\text {env }} \gamma_{0}+\gamma_{+}\left(e V / 2 k_{B}\right)$. 